\title{
SIMPLE PROOFS \\ OF BERNSTEIN-TYPE INEQUALITIES
}

\author{
R. N. MOHAPATRA, P. J. O'HARA AND R. S. RODRIGUEZ
}

(Communicated by Richard R. Goldberg)

\begin{abstract}
A polynomial identity is established by the use of Lagrange interpolation. This identity is used to obtain simple proofs of Bernstein-type inequalities, one of which is an improvement of a recent result of Frappier, Rahman, and Ruscheweyh.
\end{abstract}

Let $P_{n}$ denote the collection of complex algebraic polynomials of degree at most $n$. For $P \in P_{n}$ we will write

$$
\|P\|=\max _{|z|=1}|P(z)|
$$

A well-known result, of $\mathrm{S}$. Bernstein (for references see [11]) states that if $P \in P_{n}$ then $\left\|P^{\prime}\right\| \leq n\|P\|$.

The following theorems are three of the many improvements of Bernstein's inequality that are known.

THEOREM A. If $P \in P_{n}$ and $\widetilde{P}(z)=z^{n} \overline{P(1 / \bar{z})}$, then

$$
\max _{|z|=1}\left[\left|\widetilde{P}^{\prime}(z)\right|+\left|P^{\prime}(z)\right|\right]=n\|P\| \text {. }
$$

THEOREM B. If $P \in P_{n}$ and $z_{1}, \ldots, z_{2 n}$ are any $2 n$ equally spaced points on the unit circle, then $\left\|P^{\prime}\right\| \leq n \max _{1 \leq k \leq 2 n}\left|P\left(z_{k}\right)\right|$.

THEOREM C. If $P \in P_{n}$, then $\left\|P^{\prime}\right\| \leq n\|\operatorname{Re}(P)\|$.

In establishing the equality in Theorem A, the nontrivial part is to prove that the left side is less than or equal to the right side. This inequality appears in [4 and 8] and generalizations of this inequality can be found in [1 and 2]. As noted in [5] an immediate corollary of Theorem $\mathrm{A}$ is the fact that if $P$ is self-inversive (i.e., $\widetilde{P}=u P$ for some $u$ with $|u|=1$ ) then $\left\|P^{\prime}\right\|=(n / 2)\|P\|$ (also proved in [3, 7 and 10]). Theorem $\mathrm{B}$ appears in [1] where the authors also show that $2 n$ is the smallest number of equally spaced points that can be used. Theorem $\mathrm{C}$ is due to G. Szegö [12] and later proofs can be found in [1, 4 and 9].

The purpose of this paper is to show that Theorems A, B and C are immediate corollaries of an interesting identity, which itself is elementary to prove. Moreover this identity will yield the following improved version of Theorem B.

Received by the editors December 11, 1986.

1980 Mathematics Subject Classification (1985 Revision). Primary 41A17; Secondary 30C10. 
THEOREM $\mathrm{B}^{\prime}$. Let $z_{1}, \ldots, z_{2 n}$ be any $2 n$ equally spaced points on the unit circle, say $z_{k}=u e^{k \pi i / n},|u|=1,1 \leq k \leq 2 n$. If $P \in P_{n}$, then

$$
\left\|P^{\prime}\right\| \leq \frac{n}{2}\left[\max _{k \text { odd }}\left|P\left(z_{k}\right)\right|+\max _{k \text { even }}\left|P\left(z_{k}\right)\right|\right] .
$$

As an example of a case comparing Theorems B and $\mathrm{B}^{\prime}$, consider $P(z)=z^{n}-1$. With $z_{k}$ as described above it is clear that $P\left(z_{k}\right)=u^{n}-1$ for $k$ even and $P\left(z_{k}\right)=$ $-\left(u^{n}+1\right)$ for $k$ odd. Therefore

$$
\max _{1 \leq k \leq 2 n}\left|P\left(z_{k}\right)\right|=\max \left\{\left|u^{n}-1\right|,\left|u^{n}+1\right|\right\}:=f(u),
$$

and

$$
\frac{1}{2}\left[\max _{k \text { odd }}\left|P\left(z_{k}\right)\right|+\max _{k \text { even }}\left|P\left(z_{k}\right)\right|\right]=\frac{1}{2}\left[\left|u^{n}+1\right|+\left|u^{n}-1\right|\right]:=g(u) .
$$

It is easy to show that $g(u) \leq \sqrt{2} \leq f(u)$. Moreover when $u^{n}= \pm 1$ then $g(u)=1$ and hence for this special $P$ the inequality in Theorem $\mathrm{B}^{\prime}$ is sharp.

The identity which is central to this paper was motivated by the methods in [6]. It is stated in the following theorem.

THEOREM. Suppose $\lambda$ is any complex number with $|\lambda|=1$ and let $t_{1}, \ldots, t_{n}$ be the $n$th roots of $\lambda$. If $P \in P_{n}$ then for all $z$ with $|z|=1$,

$$
n P(z)-z P^{\prime}(z)+\frac{\lambda}{z^{n-1}} P^{\prime}(z)=\frac{1}{n} \sum_{k=1}^{n} P\left(t_{k}\right)\left|\frac{z^{n}-\lambda}{z-t_{k}}\right|^{2}
$$

and

$$
\frac{1}{n} \sum_{k=1}^{n}\left|\frac{z^{n}-\lambda}{z-t_{k}}\right|^{2}=n .
$$

PROOF. Suppose $t_{1}, \ldots, t_{n}$ are any $n$ distinct complex numbers and let $\omega(z)=$ $\left(z-t_{1}\right) \cdots\left(z-t_{n}\right)$. Note that if $P(z)=a_{n} z^{n}+\cdots+a_{0}$, then, regardless of whether or not $a_{n}=0$, the polynomial defined by $P(z)-a_{n} \omega(z)$ is in $P_{n-1}$. Therefore by using the Lagrange interpolation formula we can write

$$
P(z)-a_{n} \omega(z)=\sum_{k=1}^{n} P\left(t_{k}\right) \frac{\omega(z)}{\omega^{\prime}\left(t_{k}\right)\left(z-t_{k}\right)}
$$

If we divide this last identity by $\omega(z)$ and then differentiate we find that

$$
P(z) \omega^{\prime}(z)-P^{\prime}(z) \omega(z)=\sum_{k=1}^{n} P\left(t_{k}\right) \frac{\omega^{2}(z)}{\omega^{\prime}\left(t_{k}\right)\left(z-t_{k}\right)^{2}} .
$$

In particular when $t_{1}, \ldots, t_{n}$ are the $n$th roots of $\lambda$, so that $\omega(z)=z^{n}-\lambda$, then (3) becomes

$$
n z^{n-1} P(z)-\left(z^{n}-\lambda\right) P^{\prime}(z)=\frac{1}{\lambda n} \sum_{k=1}^{n} P\left(t_{k}\right)\left(\frac{z^{n}-\lambda}{z-t_{k}}\right)^{2} t_{k} .
$$

Note that if $|u|=|v|=1$ then $(u-v)^{2}=-|u-v|^{2} u v$. Therefore if $|z|=|\lambda|=1$ then

$$
\left(\frac{z^{n}-\lambda}{z-t_{k}}\right)^{2} t_{k}=\left|\frac{z^{n}-\lambda}{z-t_{k}}\right|^{2} \lambda z^{n-1}
$$

and (4) can be rewritten to obtain (1). To derive (2) use (1) with $P(z)=z^{n}$. 
COROLlARY. Suppose $\lambda$ is any complex number with $|\lambda|=1$. Let $t_{1}, \ldots, t_{n}$ be the $n$th roots of $\lambda$ and $s_{1}, \ldots, s_{n}$ be the $n$th roots of $-\lambda$. If $P \in P_{n}$, then for all $z$ with $|z|=1$

$$
\frac{2 \lambda}{z^{n-1}} P^{\prime}(z)=\frac{1}{n} \sum_{k=1}^{n} P\left(t_{k}\right)\left|\frac{z^{n}-\lambda}{z-t_{k}}\right|^{2}-\frac{1}{n} \sum_{k=1}^{n} P\left(s_{k}\right)\left|\frac{z^{n}+\lambda}{z-s_{k}}\right|^{2} .
$$

PROOF. Replace $\lambda$ by $-\lambda$ in (1) and subtract the resulting identity from (1).

We can now prove Theorems $\mathrm{A}, \mathrm{B}^{\prime}$ and $\mathrm{C}$.

PROOF OF THEOREM A. It follows from (1) and (2) that for all $\lambda, z$ with $|\lambda|=|z|=1$,

$$
\left\|\left[n P(z)-z P^{\prime}(z)\right]+\left(\lambda / z^{n-1}\right) P^{\prime}(z) \mid \leq n\right\| P \| .
$$

For each $z,|z|=1$, we can choose a corresponding $\lambda,|\lambda|=1$, so that the left side of this inequality becomes $\left|n P(z)-z P^{\prime}(z)\right|+\left|P^{\prime}(z)\right|$. Thus it follows that

$$
\max _{|z|=1}\left[\left|n P(z)-z P^{\prime}(z)\right|+\left|P^{\prime}(z)\right|\right] \leq n\|P\| .
$$

The reverse inequality follows easily since for $|z|=1$,

$$
|n P(z)|=\left|n P(z)-z P^{\prime}(z)+z P^{\prime}(z)\right| \leq\left|n P(z)-z P^{\prime}(z)\right|+\left|P^{\prime}(z)\right| .
$$

Therefore we have that

$$
\max _{|z|=1}\left[\left|n P(z)-z P^{\prime}(z)\right|+\left|P^{\prime}(z)\right|\right]=n\|P\| .
$$

This establishes Theorem A since it is easy to show that $\left|\widetilde{P}^{\prime}(z)\right|=\left|n P(z)-z P^{\prime}(z)\right|$ for $|z|=1$.

PROOF OF THEOREM B'. With $z_{k}=u e^{k \pi i / n},|u|=1,1 \leq k \leq 2 n$, let $t_{k}=z_{2 k}$ and $s_{k}=z_{2 k-1}, 1 \leq k \leq n$. Note that $t_{k}^{n}=u^{n}$ and $s_{k}^{n}=-u^{n}, 1 \leq k \leq n$. Theorem $\mathrm{B}^{\prime}$ is obtained easily by using (5) with $\lambda=u^{n}$ and applying the triangle inequality along with (2).

PROOF OF THEOREM C. Equate real parts in (5) and use the triangle inequality along with (2) to obtain

$$
2\left|\operatorname{Re}\left[\left(\lambda / z^{n-1}\right) P^{\prime}(z)\right]\right| \leq n\|\operatorname{Re}(P)\|+n\|\operatorname{Re}(P)\|, \quad|\lambda|=|z|=1 .
$$

Choose $z_{0}$ so that $\left|P^{\prime}\left(z_{0}\right)\right|=\left\|P^{\prime}\right\|$ and write $P^{\prime}\left(z_{0}\right)=\varepsilon\left\|P^{\prime}\right\|,|\varepsilon|=1$. Theorem $\mathrm{C}$ now follows from the last inequality by putting $z=z_{0}$ and $\lambda=z_{0}^{n-1} / \varepsilon$.

\section{REFERENCES}

1. C. Frappier, Q. I. Rahman and St. Ruscheweyh, New inequalities for polynomials, Trans. Amer. Math. Soc. 288 (1985), 69-99.

2. N. K. Govil and Q. I. Rahman, Functions of exponential type not vanishing in a half-plane and related polynomials, Trans. Amer. Math. Soc. 137 (1969), 501-517.

3. N. K. Govil, On the derivative of a polynomial, Proc. Amer. Math. Soc. 41 (1973), 543-546.

4. M. A. Malik, On the derivative of a polynomial, J. London Math. Soc. 1 (1969), 57-60.

5. __ An integral estimate for polynomials, Proc. Amer. Math. Soc. 91 (1984), 281-284.

6. P. J. O'Hara, Another proof of Bernstein's theorem, Amer. Math. Monthly 80 (1973), 673-674.

7. P. J. O'Hara and R. S. Rodriguez, Some properties of self-inversive polynomials, Proc. Amer. Math. Soc. 44 (1974), 331-335.

8. Q. I. Rahman, Applications of functional analysis to extremal problems for polynomials, Sém. Math. Supérieures, Presses Univ. Montréal, 1967. 
9. W. W. Rogosinski, Extremum problems for polynomials and trigonometrical polynomials, J. London Math. Soc. 29 (1954), 259-275.

10. E. B. Saff and T. Sheil-Small, Coefficient and integral mean estimates for algebraic and trigonometric polynomials with restricted zeros, J. London Math. Soc. 9 (1974), 16-22.

11. A. C. Schaeffer, Inequalities of A. Markoff and S. Bernstein for polynomials and related functions, Bull. Amer. Math. Soc. 47 (1941), 565-579.

12. G. Szegö, Über einen Satz des Herrn Serge Bernstein, Schriften Königsberger Gelehrten Gesellschaft 5 (1928), 59-70.

Department of Mathematics, University of Central Florida, Orlando, FLORIDA 32816 